Tôhoku Math. Journ. 39 (1987), 505-517.

\title{
A RELAXATION THEOREM FOR DIFFERENTIAL INCLUSIONS IN BANACH SPACES
}

\author{
Nikolaos S. Papageorgiou*
}

(Received May 1, 1986)

\begin{abstract}
In this paper we consider differential inclusions in a separable Banach space and we show that when the orientor field satisfies the Caratheodory conditions and is Lipschitzean with respect to a Kamke function $w(t, x)$ in the state variable, then the set of solutions of the nonconvex problem is dense in the $C_{X}(T)$-topology in the set of solutions of the convexified problem.
\end{abstract}

Introduction. For a differential inclusion $\dot{x}(t) \in F(t, x(t))$ with a nonconvex right hand side the set of solutions through an initial point is, in general, not closed (even if its sections might be). So we would like to know what is the relation of the closure of this set to the set of the solutions of the convexified problem. This problem was first considered by Ważewski [20] who introduced the notion of a quasitrajectory and proved that whenever $F(t, x)$ is continuous, every solution $\dot{x}(t) \in \operatorname{clconv} F(t, x(t))$ is the limit of a sequence of quasitrajectories of $\dot{x}(t) \in F(t, x(t))$. However such a result does not provide an estimate of the distance between a quasitrajectory and a true solution. In fact such an estimate cannot be obtained if we only assume that $F(\cdot, \cdot)$ is continuous. The additional condition needed is a Lipschitzness condition in the $x$-variable of $F(\cdot, \cdot)$. With that condition present, Filippov [5] was able to obtain the missing estimate and then prove the desired density result. A very nice presentation of those results can be found in the book of Clarke [3, pp. 115118]. Later Pliss [12] provided a counterexample which illustrated that the Lipschitzness condition cannot be omitted. A generalization of Filippov's theorem was given by Pianigiani [11]. However all these results were for $\boldsymbol{R}^{n}$. The only infinite dimensional relaxation result that we know of, is that of Tolstonogov [17, Theorem 4.3], which was stated though without a proof. Here we present another such theorem, with a different set of hypotheses. The motivation for such an infinite dimensional result comes from the optimal control theory of systems governed by an evolution equation (distributed parameter systems, see for example Ahmed-Teo [1]).

\footnotetext{
* Research supported by N.S.F. Grants D.M.S. -8403135 and D.M.S. 8602313.
} 
2. Preliminaries. Let $(\Omega, \Sigma, \mu)$ be a complete $\sigma$-finite measure space and $X$ a separable Banach space, with $X^{*}$ being its topological dual. We will use the following notations:

$$
\begin{aligned}
& P_{f(0)}(X)=\{A \cong X: \text { nonempty, closed, (convex) }\} \\
& P_{k(0)}(X)=\{A \cong X: \text { nonempty, compact, (convex) }\} .
\end{aligned}
$$

For $A \in 2^{X} \backslash\{\varnothing\}$, we set $|A|=\sup _{x \in A}\|x\|$, by $d_{A}(\cdot)$ we denote the distance function from $A$, i.e., for all $x \in X, d_{A}(x)=\inf _{a \in A}\|x-a\|$ and by $\sigma_{A}(\cdot)$ the support function of $A$ i.e., $\sigma_{A}\left(x^{*}\right)=\sup _{a \in A}\left(x^{*}, a\right)$ for all $x^{*} \in X^{*}$. Also by $\delta_{A}(\cdot)$ we will denote the indicator function of $A$, i.e., $\delta_{A}(y)=0$ if $y \in A$ and $+\infty$ if $y \notin A$.

A multifunction $F: \Omega \rightarrow P_{f}(X)$ is said to be measurable if it satisfies any of the following equivalent conditions:

(1) $\omega \rightarrow d_{F(\omega)}(x)$ is measurable for all $x \in X$.

(2) there exists a sequence $\left\{f_{n}(\cdot)\right\}_{n \geqq 1}$ of measurable functions such that $F(\omega)=\operatorname{cl}\left\{f_{n}(\omega)\right\}_{n \geqq 1}$ for all $\omega \in \Omega$ (Castaing's representation).

(3) $\operatorname{Gr} F=\{(\omega, x) \in \Omega \times X: x \in F(\omega)\} \in \Sigma \times B(X)$, where $B(X)$ is the Borel $\sigma$-field of $X$.

Any multifunction, not necessarily closed valued, satisfying (3) is said to be graph measurable.

We denote by $S_{F}^{1}$ the set of all selectors of $F(\cdot)$ that belong to the Lebesgue-Bochner space $L_{X}^{1}(\Omega)$, i.e., $S_{F}^{1}=\left\{f(\cdot) \in L_{X}^{1}(\Omega): f(\omega) \in F(\omega) \mu\right.$-a.e. $\}$ Clearly this is nonempty if $F(\cdot)$ is integrably bounded, i.e., $|F(\cdot)| \in L_{+}^{1}(\Omega)$. Using $S_{F}^{1}$ we can define a set valued integral for $F(\cdot)$ by setting $\int_{\Omega} F(\omega) d \mu(\omega)=\left\{\int_{\Omega} f(\omega) d \mu(\omega): f(\cdot) \in S_{F}^{1}\right\}$.

If $\left\{A_{n}\right\}_{n \geq 1}$ is a sequence of nonempty subsets of $X$, we define:

$$
\text { s-liminf } A_{n \rightarrow \infty}=\left\{x \in X: x=\operatorname{s}-\lim x_{n}, x_{n} \in A_{n}, n \geqq 1\right\}
$$

and

$$
\text { w-limsup } A_{n \rightarrow \infty}=\left\{x \in X: x=\mathrm{w}-\lim x_{n_{k}}, x_{n_{k}} \in A_{n_{k}}, k \geqq 1\right\} .
$$

We will say that the $A_{n}$ 's converge to $A$ in the Kuratowski-Mosco sense (denoted by $A_{n} \stackrel{\mathrm{K}-\mathrm{M}}{\longrightarrow} A$ ) if and only if w-limsup ${ }_{n \rightarrow \infty} A_{n}=A=\operatorname{s-liminf}_{n \rightarrow \infty} A_{n}$. Using this mode of set convergence, we can define a convergence for functions which is in general different from the pointwise convergence. So if $\left\{f_{n}, f\right\}_{n \geq 1} \subseteq \overline{\boldsymbol{R}}^{x}$ are proper functions, we say that $f_{n} \stackrel{\tau}{\rightarrow} f$ if and only if epif $_{n} \stackrel{\mathrm{K}-\mathrm{M}}{\longrightarrow}$ epif. For more details about the $\mathrm{K}-\mathrm{M}$ and $\tau$-convergences, we refer to Mosco [10] and Salinetti-Wets [16].

Finally if $A, B \in P_{f}(X)$, we set $h(A, B)=\sup _{x \in X}\left|d_{A}(x)-d_{B}(x)\right|$ (the 
Hausdorff metric). Also recall that by a Kamke function, we mean a function $w:[0, b] \times \boldsymbol{R}_{+} \rightarrow \boldsymbol{R}_{+}$satisfying the Caratheodory conditions (i.e., it is measurable in $t$ and continuous in $x$ ) integrably bounded on bounded subsets of $T \times \boldsymbol{R}_{+}, w(t, 0) \equiv 0$ a.e. and such that $u(t) \equiv 0$ is the only solution of the problem $u(t) \leqq \int_{0}^{t}(w(s, u(s)) d s, u(0)=0$.

In the proof of the relaxation theorem, we will need the following results, which are also interesting on their own as general results about multifunctions and so we state them in the most general from we were able to prove. Here $(\Omega, \Sigma, \mu)$ is a $\sigma$-finite measure space, with $\Sigma$ being a Souslin family (in particular $\Sigma$ may be $\mu$-complete) and $X$ is a separable Banach space.

Proposition 2.1 If $F: \Omega \rightarrow 2^{X} \backslash\{\varnothing\}$ is graph measurable and

$$
\begin{aligned}
S_{P}^{1} \neq & \varnothing \text { then for all } x^{*} \in X^{*}, \sigma\left(x^{*}, \int_{\Omega} F\right) \\
& =\sup \left\{\left(x^{*}, x\right): x \in \int_{\Omega} F\right\}=\int_{\Omega} \sigma_{F(\omega)}\left(x^{*}\right) d \mu(\omega) .
\end{aligned}
$$

Proof. Our proof follows Rockafellar [15, Theorem 3A].

From Theorem 5.10, of Wagner [19] we know that $F(\cdot)$ admits a Castaing representation $\left\{f_{n}(\cdot)\right\}_{n \geqq 1}$. Hence

$$
\sigma_{F(\omega)}\left(x^{*}\right)=\sup _{n \geqq 1}\left(x^{*}, f_{n}(\omega)\right) \Rightarrow \omega \rightarrow \sigma_{F^{\prime}(\omega)}\left(x^{*}\right) \text { is measurable. }
$$

Also since $\left(x^{*}, f(\omega)\right) \leqq \sigma_{F(\omega)}\left(x^{*}\right)$ and $\left(x^{*}, f(\cdot)\right) \in L^{1}$ for $f(\cdot) \in S_{F}^{1}$, we deduce that for all $x^{*} \in X^{*}, \omega \rightarrow \sigma_{F(\omega)}\left(x^{*}\right)$ is quasiintegrable (i.e., $\left.\left[\sigma_{F(\cdot)}\left(x^{*}\right)\right]^{-} \in L^{1}\right)$.

Directly from the definitions we can see that we always have:

$$
\sigma\left(x^{*}, \int_{\Omega} F\right) \leqq \int_{\Omega} \sigma_{F(\omega)}\left(x^{*}\right) d \mu(\omega)
$$

Fix $x^{*} \in X^{*}$ and let $\beta<\int_{\Omega} \sigma_{F(\omega)}\left(x^{*}\right) d \mu(\omega)$. Our goal is to show that there exists $\hat{f}(\cdot) \in S_{F}^{1}$ such that

$$
\beta<\int_{\Omega}\left(x^{*}, \hat{f}(\omega)\right) d \mu(\omega) .
$$

Take $\left\{\Omega_{n}\right\}_{n \geqq 1}$ monotone increasing in $\Sigma$ such that $\mu\left(\Omega_{n}\right)<\infty$ and $\Omega=$ $\cup_{n \geqq 1} \Omega_{n}$ and let $p(\cdot) \in L_{+}^{1}, p(\omega)>0$ for all $\omega \in \Omega$. For $n \geqq 1$ define:

$$
A_{n}=\left\{\omega \in \Omega: \sigma_{F(\omega)}\left(x^{*}\right) \leqq n\right\} \cap \Omega_{n} .
$$

Then set 


$$
\begin{aligned}
g_{n}(\omega) & =\sigma_{F(\omega)}\left(x^{*}\right)-\frac{1}{n} p(\omega) \text { for } \omega \in A_{n} \\
& =\left(x^{*}, f(\omega)\right)-\frac{1}{n} p(\omega) \text { for } \quad \omega \in \Omega \backslash A_{n} .
\end{aligned}
$$

Clearly $g_{n}(\cdot) \in L^{1}$ and $g_{n}(\omega) \uparrow \sigma_{F(\omega)}\left(x^{*}\right) \mu$-a.e. So we can find $n_{0} \geqq 1$ such that $\int_{\Omega} g_{n}(\omega) d \mu(\omega)>\beta$ for all $n \geqq n_{0}$. Set $g(\cdot)=g_{n_{0}}(\cdot)$. Let $R(\omega)=$ $\left\{x \in F(\omega): g(\omega) \leqq\left(x^{*}, x\right)\right\}$. Because $g(\omega)<\sigma_{F(\omega)}\left(x^{*}\right) \mu$-a.e., we see that $R(\omega) \neq \varnothing$, for all $\omega \in \Omega^{\prime}=\Omega \backslash N, \mu(N)=0$. Also Gr $\left.R\right|_{\Omega^{\prime}}=\left\{(\omega, x) \in \Omega^{\prime} \times X\right.$ : $\left.g(\omega)-\left(x^{*}, x\right) \leqq 0\right\} \cap \operatorname{Gr} F \in\left(\Sigma \cap 2^{\Omega^{\prime}}\right) \times B(X)$. Hence we can apply Aumann's selection theorem to find $h: \Omega^{\prime} \rightarrow X$ measurable such that $h(\omega) \in R(\omega)$ for all $\omega \in \Omega^{\prime}$. Extend $h(\cdot)$ on $\Omega$. Then $h(\cdot)$ becomes a measurable selector of $F(\cdot)$ but it is not necessarily in $L_{X}^{1}(\Omega)$. Furthermore note that

$$
\beta<\int_{\Omega}\left(x^{*}, h(\omega)\right) d \mu(\omega) \text {. }
$$

Next let $B_{n}=\left\{\omega \in \Omega: \| h(\omega \| \leqq n\} \cap A_{n}\right.$ and define:

$$
\hat{f}_{n}(\cdot)=\chi_{B_{n}}(\cdot) h(\cdot)+\chi_{\Omega \backslash B_{n}}(\cdot) f(\cdot) \text {. }
$$

Clearly $\left\{f_{n}(\cdot)\right\}_{n \geqq 1} \subseteq S_{F}^{1}$ and

$$
\begin{aligned}
\int_{\Omega}\left(x^{*}, \hat{f}_{n}(\omega)\right) d \mu(\omega) & =\int_{B_{n}}\left(x^{*}, h(\omega)\right) d \mu(\omega)+\int_{\Omega \backslash B_{n}}\left(x^{*}, f(\omega)\right) d \mu(\omega) \\
& \geqq \int_{B_{n}} g(\omega) d \mu(\omega)+\int_{\Omega \backslash B_{n}}\left(x^{*}, f(\omega)\right) d \mu(\omega) \\
& =\int_{\Omega} g(\omega) d \mu(\omega)+\int_{\Omega \backslash B_{n}}\left[\left(x^{*}, f(\omega)-g(\omega)\right)\right] d \mu(w) .
\end{aligned}
$$

Recalling that $\int_{\Omega} g(\omega) d \mu(\omega)>\beta$ and that $\mu\left(\Omega \backslash B_{n}\right) \downarrow 0$ we finally have that for large enough $n$

$$
\int_{\Omega}\left(x^{*}, \hat{f}_{n}(\omega)\right) d \mu(\omega)>\beta
$$

Since $\hat{f}_{n}(\cdot) \in S_{F}^{1}$, the proof is finished.

q.e.d.

The next proposition establishes the convexity of the set valued integral. Our result generalizes the corresponding theorem for $\boldsymbol{R}^{n}$-valued multifunctions (see for example Theorem 7.1.6 in Klein-Thompson [7]). So assume that $(\Omega, \Sigma, \mu)$ is nonatomic, $\sigma$-finite, with $\Sigma$ a Souslin family and $X$ is a separable Banach space.

Proposition 2.2. If $F: \Omega \rightarrow 2^{x} \backslash\{\varnothing\}$ is graph measurable and $S_{F}^{1} \neq \varnothing$ then $\mathrm{cl} \int_{\Omega} F(\omega) d \mu(\omega)$ is convex. 
Proof. Let $x_{1}, x_{2} \in \operatorname{cl} \int_{\Omega} F(\omega) d \mu(\omega)$. Then given $\varepsilon>0$ there exist $f_{1}(\cdot)$, $f_{2}(\cdot) \in S_{F}^{1}$ such that $\left\|x_{1}-\int_{0}^{D} f_{1}(\omega) d \mu(\omega)\right\|<\varepsilon / 2$ and $\left\|x_{2}-\int_{0} f_{2}(\omega) d \mu(\omega)\right\|<$ $\varepsilon / 2$. Consider the vector valued measure $r: \Sigma \rightarrow X \times X$ defined by

$$
r(A)=\left(\int_{A} f_{1}(\omega) d \mu(\omega), \int_{A} f_{2}(\omega) d \mu(\omega)\right) .
$$

Because $\mu(\cdot)$ is nonatomic, Corollary 1 of Kluvanek-Knowles [8, p. 98] tells us that the norm closure of the range of $r(\cdot)$ is convex. Note that $r(\varnothing)=(0,0)$ and $r(\Omega)=\left(\int_{\Omega} f_{1}(\omega) d \mu(\omega), \int_{\Omega} f_{2}(\omega) d \mu(\omega)\right)$. Hence for $\lambda \in(0,1)$, there exists $A \in \Sigma$ such that

$$
\begin{aligned}
& \|r(A)-\lambda r(\Omega)\|<\varepsilon / 4 \text { and }\|r(\Omega \backslash A)-(1-\lambda) r(\Omega)\|<\varepsilon / 4 \\
& \quad \Rightarrow\left\|\int_{A} f_{i}(\omega) d \mu(\omega)-\lambda \int_{\Omega} f_{i}(\omega) d \mu(\omega)\right\|<\varepsilon / 4
\end{aligned}
$$

and

$$
\left\|\int_{\Omega \backslash A} f_{i}(\omega) d \mu(\omega)-(1-\lambda) \int_{\Omega} f_{i}(\omega) d \mu(\omega)\right\|<\varepsilon / 4
$$

for $i=1,2$. Set $f=\chi_{A} f_{1}+\chi_{Q \backslash A} f_{2}$. Clearly $f \in S_{F}^{1}$. Then we have:

$$
\begin{aligned}
\| \lambda x_{1}+ & (1-\lambda) x_{2}-\int_{\Omega} f(\omega) d \mu(\omega) \| \\
\leqq & \left\|\lambda x_{1}-\lambda \int_{\Omega} f_{1}(\omega) d \mu(\omega)\right\|+\left\|\lambda \int_{\Omega} f_{1}(\omega) d \mu(\omega)-\int_{A} f_{1}(\omega) d \mu(\omega)\right\| \\
& +\left\|(1-\lambda) x_{2}-(1-\lambda) \int_{\Omega} f_{2}(\omega) d \mu(\omega)\right\| \\
& +\left\|(1-\lambda) \int_{\Omega} f_{2}(\omega) d \mu(\omega)-\int_{\Omega \backslash A} f_{2}(\omega) d \mu(\omega)\right\| \\
< & \lambda \varepsilon / 2+\varepsilon / 4+(1-\lambda) \varepsilon / 2+\varepsilon / 4=\varepsilon .
\end{aligned}
$$

So indeed $\mathrm{cl} \int_{\Omega} F(\omega) d \mu(\omega)$ is convex.

q.e.d.

Using the previous two propositions we can have the following interesting property of the set valued integral. Let $(\Omega, \Sigma, \mu)$ be a nonatomic, complete, $\sigma$-finite measure space, while $X$ is still a separable Banach space.

Proposition 2.3. If $F: \Omega \rightarrow 2^{X} \backslash\{\varnothing\}$ is graph measurable and $S_{F}^{1} \neq \varnothing$ then $\mathrm{cl} \int_{\Omega} F(\omega) d \mu(\omega)=\mathrm{cl} \int_{\Omega} \operatorname{clconv} F(\omega) d \mu(\omega)$.

Proof. For all $x^{*} \in X^{*}$, using Proposition 2.1 we have: 


$$
\sigma\left(x^{*}, \operatorname{cl} \int_{\Omega} F\right)=\sigma\left(x^{*}, \int_{\Omega} F\right)=\int_{\Omega} \sigma_{F(\omega)}\left(x^{*}\right) d \mu(\omega)=\int_{\Omega} \sigma\left(x^{*}, \operatorname{clconv} F(\omega)\right) d \mu(\omega) .
$$

Using Theorem III-40 of Castaing-Valadier [2], we have that $\omega \rightarrow$ clconv $F(\omega)$ is measurable. So a new application of Proposition 2.1. gives us:

$$
\begin{gathered}
\int_{\Omega} \sigma\left(x^{*}, \operatorname{clconv} F(\omega)\right) d \mu(\omega)=\sigma\left(x^{*}, \int_{\Omega} \operatorname{clconv} F\right) \\
\Rightarrow \sigma\left(x^{*}, \operatorname{cl} \int_{\Omega} F\right)=\sigma\left(x^{*}, \operatorname{cl} \int_{\Omega} \operatorname{clconv} F\right) .
\end{gathered}
$$

For all $x^{*} \in X^{*}$. But from Proposition 2.2. we know that $\mathrm{cl} \int_{\Omega} F$ is convex. So finally we have:

$$
\mathrm{cl} \int_{\Omega} F(\omega) d \mu(\omega)=\operatorname{cl} \int_{\Omega} \operatorname{clconv} F(\omega) d \mu(\omega) .
$$

q.e.d.

REMARK. If $F: \Omega \rightarrow 2^{X}$ is integrably bounded and has nonempty, w-compact, convex values, then we have: $\operatorname{cl} \int_{\Omega} F(\omega) d \mu(\omega)=\int_{\Omega} \operatorname{clconv} F(\omega) d \mu(\omega)$ (see the Corollary to Proposition 3.1. in [13]).

3. Main theorem. Let $T=[0, b]$ be a closed, bounded subinterval in $\boldsymbol{R}_{+}$and let $X$ be a separable, Banach space.

We will consider the following two multivalued Cauchy problems.

$$
\left\{\begin{array}{l}
\dot{x}(t) \in F(t, x(t)) \\
x(0)=x_{0}
\end{array}\right.
$$

and

$$
\left\{\begin{array}{l}
\dot{x}(t) \in \overline{\operatorname{conv}} F(t, x(t)) \\
x(0)=x_{0}
\end{array}\right.
$$

By a solution of $\left({ }^{*}\right)\left(\right.$ resp. of $\left(^{* *}\right)$ ) we understand an absolutely continuous function $x(\cdot)$ satisfying $\left({ }^{*}\right)\left(\right.$ resp. $\left({ }^{* *}\right)$ ) almost everywhere. We will denote the solution set of $\left(^{*}\right)$ by $P$ and the solution set of $\left(^{* *}\right)$ by $P_{c}$. The next theorem gives us a relation between those two solution sets. We remark that we could have stated the theorem for a locally defined orientor field i.e., $\operatorname{dom} F=T \times B_{r}\left(x_{0}\right)$, where $B_{r}\left(x_{0}\right)=\{x \in X$ : $\left.\left\|x-x_{0}\right\|<r\right\}$, in which case the solutions are, in general, defined on a subinterval of $T$. But in order to simplify an already lengthy and complicated proof, we have decided to work with a globally defined orientor field, i.e., $\operatorname{dom} F=T \times X$. Also note that the conditions that we need in order to prove our relaxation theorem also guarantee the nonemptiness 
of the solution sets (see Theorem 4.2 of [14]).

THEOREM 3.1. If $F: T \times X \rightarrow P_{k}(X)$ is a multifunction such that

(1) for all $x \in X, t \rightarrow F(t, x)$ is measurable and $F(t, x) \subseteq G(t)$ a.e., where $G: T \rightarrow P_{k c}(X)$ is integrably bounded;

(2) for all $(x, y) \in X \times X$ we have $h(F(t, x), F(t, y)) \leqq w(t,\|x-y\|)$ a.e., where $w(\cdot, \cdot)$ is a Kamke function.

Then $P_{c}=\bar{P}$ where the closure is taken in $C_{X}(T)$.

Proof. Because the proof is lengthy and complicated, in order to assist the reader in following our reasoning, we will divide it into steps.

Step 1. Given $x(\cdot) \in P_{c}$ and $\varepsilon>0$ we will find $v(\cdot) \in C_{X}(T)$ such that $v(0)=x_{0}, \dot{v}(t) \in F(t, x(t))$ a.e., and $\|v(t)-x(t)\|<\varepsilon$ for all $t \in T$.

Since $x(\cdot) \in P_{c}$, for all $t \in T$ we have that

$$
x(t) \in x_{0}+\int_{0}^{t} \operatorname{clconv} F(s, x(s)) d s .
$$

From the corollary of Proposition 3.1 of [13] we know that $\int_{0}^{t}$ clconv $F(s, x(s)) d s \in P_{f_{c}}(X)$. Also from Proposition 2.3 we have that

$$
\int_{0}^{t} \operatorname{clconv} F(s, x(s)) d s=\operatorname{cl} \int_{0}^{t} F(s, x(s)) d s .
$$

So for all $t \in T$, we have:

$$
x(t) \in x_{0}+\operatorname{cl} \int_{0}^{t} F(s, x(s)) d s .
$$

Let $\left\{T_{k}\right\}_{k=1}^{n}$ be a subdivision of $T$ into $n$ disjoint intervals such that $\int_{T_{k}}|G(s)| d s<\varepsilon / 3$. Also let $z_{k}(\cdot) \in S_{F(\cdot, x(\cdot))}^{1}$ such that

$$
\left\|\int_{T_{k}}\left(\dot{x}(s)-z_{k}(s)\right) d s\right\|<\varepsilon / 3 n .
$$

Define $z(\cdot) \in S_{F(\cdot, x(\cdot))}^{1}$ by setting $z(t)=z_{k}(t)$ for all $t \in T_{k}, k=1, \cdots, n$. Then set $v(t)=x_{0}+\int_{0}^{t} z(s) d s$. Suppose $T_{k} \subseteq[0, t]$ for $k=1, \cdots, m \leqq n$. Then we have:

$$
\begin{aligned}
\| v(t) & -x(t)\|=\| \sum_{k=1}^{m} \int_{T_{k}}(z(s)-\dot{x}(s)) d s+\int_{[0, t] \cap T_{m+1}}(z(s)-\dot{x}(s)) d s \| \\
& \leqq \sum_{k=1}^{m}\left\|\int_{T_{k}}\left(z_{k}(s)-\dot{x}(s)\right) d s\right\|+2 \int_{T_{m+1}}|G(s)| d s<n(\varepsilon / 3 n)+2(\varepsilon / 3)=\varepsilon .
\end{aligned}
$$

This completes the proof of Step 1. that

So from this step we know that we can find $\left\{v_{n}(\cdot)\right\}_{n \geqq 1} \subseteq C_{X}(T)$ such 


$$
v_{n}(0)=x_{0} \cdot \dot{v}_{n}(t) \in F(t, x(t)) \text { a.e. }
$$

and

$$
\left\|v_{n}(t)-x(t)\right\|<\frac{1}{n} \text { for all } t \in T .
$$

Step 2. $W=\left\{y(\cdot) \in C_{X}(T): y(t)=x_{0}+\int_{0}^{t} g(s) d s, t \in T, g \in S_{G}^{1}\right\}$ is a compact subset of $C_{X}(T)$.

Let $y(\cdot) \in W$ and $t, t^{\prime} \in T, t \leqq t^{\prime}$. Then for some $g(\cdot) \in S_{G}^{1}$ we have:

$$
\left\|y\left(t^{\prime}\right)-y(t)\right\|=\left\|x_{0}+\int_{0}^{t^{\prime}} g(s) d s-x_{0}-\int_{0}^{t} g(s) d s\right\| \leqq \int_{t}^{t^{\prime}}\|g(s)\| d s \leqq \int_{t}^{t^{\prime}}|G(s)| d s,
$$

which shows that $W$ is equicontinuous.

Also for all $y(\cdot) \in W$ and all $t \in T$ we have that

$$
y(t) \in x_{0}+\int_{0}^{t} G(s) d s .
$$

Since $G(\cdot)$ is $P_{k c}(X)$-valued, because of the Radström embedding (see Theorem 17.2.1, of Klein-Thompson [7, p. 189]). $G(\cdot)$ can be viewed as an $\hat{X}$ valued, integrable, single valued function, where $\hat{X}$ is a separable Banach space (in fact $\hat{X}=C\left(B_{1}^{*}\right)$, where $B_{1}^{*}$ is the unit ball of the dual of $X$ ). Then $\int_{0}^{t} G(s) d s$ can be viewed as a Bochner integral and so finally $\int_{0}^{t} G(s) d s \in$ $P_{k c}(X)$. Hence for all $t \in T, \operatorname{cl}\{y(t)\}_{y(\cdot) \in W} \in P_{k}(X)$. Finally let $\left\{y_{n}(\cdot)\right\}_{n \geqq 1} \subseteq W$ and assume that $y_{n}(\cdot) \stackrel{C_{X}(T)}{\longrightarrow} y(\cdot)$. Then for all $n \geqq 1$ and all $t \in T$ we have:

$$
y_{n}(t)=x_{0}+\int_{0}^{t} g_{n}(s) d s,
$$

where $g_{n}(\cdot) \in S_{G}^{1}$. But from Proposition 3.1 of [13] we know that $S_{G}^{1}$ is w-compact in $L_{X}^{1}(T)$ and by the Eberlein-Smulian theorem it is sequentially w-compact. Hence by passing to a subsequence, if necessary, we may assume that $g_{n}(\cdot) \stackrel{\mathrm{w}-L_{X}^{1}}{\longrightarrow} g(\cdot)$ as $n \rightarrow \infty \Rightarrow \int_{0}^{t} g_{n}(s) d s \stackrel{\mathrm{w}}{\rightarrow} \int_{0}^{t} g(s) d s \Rightarrow y_{n}(t)=x_{0}+$ $\int_{0}^{t} g_{n}(s) d s \stackrel{\mathrm{w}}{\rightarrow} x_{0}+\int_{0}^{t} g(s) d s \Rightarrow y(t)=x_{0}+\int_{0}^{t} g(s) d s$ for all $t \in T \Rightarrow y(\cdot) \in W \Longrightarrow W$ is closed in $C_{X}(T)$. So invoking the Arzela-Ascoli theorem we deduce that $W$ is a compact subset of $C_{X}(T)$.

Next let $R_{n}: T \times W \rightarrow P_{k}(X)$ be defined as follows:

$$
R_{n}(t, y)=\left\{u \in F(t, y(t)):\left\|\dot{v}_{n}(t)-u\right\| \leqq d\left(\dot{v}_{n}(t), F(t, y(t))\right)+\frac{1}{n}\right.
$$

where $\left\{v_{n}(\cdot)\right\}_{n \geqq 1}$ are the function obtained in Step 1 .

Step 3. $\quad(t, y) \rightarrow R_{n}(t, y)$ in graph measurable for every $n \geqq 1$.

Consider the map $\psi_{n}: T \times W \times X \rightarrow R$ defined by 


$$
\psi_{n}(t, y(\cdot), u)=\left\|\dot{v}_{n}(t)-u\right\|-d\left(\dot{v}_{n}(t), F(t, y(t))\right) \text {. }
$$

Note that because of our hypotheses on $F(\cdot, \cdot)$ for all $z \in X(t, y) \rightarrow$ $d(z, F(t, y))$ is a Caratheodory function $\Rightarrow t \rightarrow d(z, F(t, y(t))$ is measurable. Then for the same reason $t \rightarrow d\left(\dot{v}_{n}(t), F(t, y(t))\right)$ is measurable. So we deduce that $t \rightarrow \psi_{n}(t, y(\cdot), u)$ is measurable. Furthermore, using the fact that $F(t, \cdot)$ is Hausdorff continuous for all $t \in T$, we can easily see that $(y(\cdot), u) \rightarrow \psi_{n}(t, y(\cdot), u)$ is continuous. Thus $(t, y(\cdot), u) \rightarrow \psi_{n}(t, y(\cdot), u)$ is a Caratheodory function, hence it is jointly measurable. Therefore we have that:

$$
\begin{aligned}
\{(t, & \left.y(\cdot), u) \in T \times W \times X: \psi(t, y(\cdot), u) \leqq \frac{1}{n}\right\} \in \Sigma \times B(W) \times B(X) \\
& \Rightarrow \operatorname{Gr} R_{n} \in \Sigma \times B(W) \times B(X) \\
& \Rightarrow R_{n}(\cdot, \cdot) \text { is graph measurable, } n \geqq 1
\end{aligned}
$$

Step 4. For every $n \geqq 1, y(\cdot) \rightarrow R_{n}(t, y)$ is continuous for the $\mathrm{K}-\mathrm{M}$ convergence for almost all $t \in T$.

Fix $n \geqq 1$ and let $y_{m}(\cdot) \stackrel{W}{\rightarrow} y(\cdot)$ as $m \rightarrow \infty$. Define

$$
z_{m}(t, u)=\left\|\dot{v}_{n}(t)-u\right\|-d\left(\dot{v}_{n}(t), F\left(t, y_{m}(t)\right)\right)+\delta\left(u, F\left(t, y_{m}(t)\right)\right)
$$

and

$$
z(t, u)=\left\|\dot{v}_{n}(t)-u\right\|-d\left(\dot{v}_{n}(t), F(t, y(t))\right)+\delta(u, F(t, y(t))) .
$$

Observe that $d\left(\dot{v}_{n}(t), F\left(t, y_{m}(t)\right)\right) \rightarrow d\left(\dot{v}_{n}(t), F(t, y(t))\right)$ as $m \rightarrow \infty$, for all $t \in$ $T \backslash N_{n}^{\prime}, \lambda\left(N_{n}^{\prime}\right)=0$. Note that $N_{n}^{\prime}$ is independent of the sequence $\left\{y_{m}(\cdot)\right\}_{m \geqq 1}$, and is the union of the exceptional sets postulated from the inequality of the hypothesis 2 and the fact that $\dot{v}_{n}(\cdot)$ exists almost everywhere. Also since $F\left(t, y_{m}(t)\right) \stackrel{h}{\rightarrow} F(t, y(t))$ for all $t \in T$ and $F(t, z) \subseteq G(t)$ a.e., it is easy to see that $F\left(t, y_{m}(t)\right) \stackrel{\mathrm{K}-\mathrm{M}}{\longrightarrow} F(t, y(t))$ a.e. So from Mosco [10], we get that

$$
\delta\left(\cdot, F\left(t, y_{m}(t)\right)\right) \stackrel{\tau}{\rightarrow} \delta(\cdot, F(t, y(t)))
$$

for all $t \in T \backslash N^{\prime \prime}, \lambda\left(N^{\prime \prime}\right)=0$. Again $N^{\prime \prime}$ is independent of $\left\{y_{m}(\cdot)\right\}_{m \geqq 1}$ and is the null set, outside of which we have $G(\cdot)$ bounding $F(\cdot, \cdot)$. Let $N_{n}=N_{n}^{\prime} \cup N^{\prime \prime}$. Then $\lambda\left(N_{n}\right)=0$. Let $t \in T \backslash N_{n}$. From Lemma 1.10 of Mosco [10] (see also Lemma 1.1 of Salinetti-Wets [16]) we know that we can find $\left\{u_{m}\right\}_{m \geqq 1} \subseteq X, u_{m} \stackrel{\mathrm{s}}{\rightarrow} u$ such that

$$
\begin{gathered}
\underset{m \rightarrow \infty}{\limsup } \delta\left(u_{m}, F\left(t, y_{m}(t)\right)\right) \leqq \delta(u, F(t, y(t))) \\
\Rightarrow \limsup _{m \rightarrow \infty} z_{m}\left(t, u_{m}\right) \leqq z(t, u) .
\end{gathered}
$$


Also for any $\left\{u_{m}\right\}_{m \geqq 1} \subseteq X$, such that $u_{m} \stackrel{\mathrm{w}}{\rightarrow} u$ using once more the lemma of Mosco [10] and the fact that in a Banach space the norm is weaklylower semicontinuous we have that

and

$$
\delta(u, F(t, y(t))) \leqq \liminf _{m \rightarrow \infty} \delta\left(u_{m}, F\left(t, y_{m}(t)\right)\right)
$$

$$
\left\|\dot{v}_{n}(t)-u\right\| \leqq \liminf _{m \rightarrow \infty}\left\|\dot{v}_{n}(t)-u_{m}\right\|
$$

Combining those two facts we get that

$$
z(t, u) \leqq \liminf _{m \rightarrow \infty} z_{m}\left(t, u_{m}\right) \text {. }
$$

From (1), (2) and Lemma 1.10 of Mosco [10], we deduce that for all $t \in T \backslash N_{n}, \lambda\left(N_{n}\right)=0$, we have:

$$
z_{m}(t, \cdot) \stackrel{\tau}{\rightarrow} z(t, \cdot)
$$

Let $N=\cup_{n \geq 1} N_{n}$. Invoking Corollary 4 of Wets [21, p. 262] we have that for all $n \geqq 1$ and all $t \in T \backslash N, \lambda(N)=0$,

$$
L_{m}^{n}(t) \stackrel{\mathrm{K}-\mathrm{M}}{\longrightarrow} L(t) \text { as } m \rightarrow \infty,
$$

where $L_{m}^{n}(t)=\left\{u \in X: z_{m}(t, u) \leqq 1 / n\right\}$ and $L^{n}(t)=\{u \in X: z(t, u) \leqq 1 / n\}$. But note that $L_{m}^{n}(t)=R_{n}\left(t, y_{m}\right)$ and $L^{n}(t)=R_{n}(t, y)$. So for all $n \geqq 1$ we have that

$$
R_{n}\left(t, y_{m}\right) \stackrel{\mathrm{K}-\mathrm{M}}{\longrightarrow} R_{n}(t, y)
$$

for all $t \in T \backslash N, \lambda(N)=0$. By redefining $R_{n}(\cdot, \cdot)$ on the set $N$, we may assume that $y(\cdot) \rightarrow R_{n}(t, y)$ is continuous for the $\mathrm{K}-\mathrm{M}$ convergence for all $t \in T, n \geqq 1$. This in particular implies that for all $n \geqq 1$ and all $t \in T$, $y(\cdot) \rightarrow R_{n}(t, y)$ is lower semicontinuous.

Consider the multifunction $H_{n}: W \rightarrow 2^{L^{1(T, X)}} \backslash\{\varnothing\}$ defined by $H_{n}(y)=$ $S_{R_{n}(\cdot, y)}^{1}, n \geqq 1$. Since $R_{n}(\cdot, y)$ is graph measurable and $R_{n}(t, y) \subseteq F(t, y(t))$, we deduce that $H_{n}(\cdot)$ is $P_{f}\left(L^{1}(T, X)\right)$-valued, $n \geqq 1$.

Step 5. For all $n \geqq 1, H_{n}(\cdot)$ is lower semicontinuous.

Let $y_{m}(\cdot) \stackrel{\mathrm{w}}{\rightarrow} y(\cdot)$. Then for every $q(\cdot) \in L^{1}(T, X)$ we have:

$$
\begin{aligned}
d\left(q, S_{R_{n}\left(\cdot, y_{m}\right)}^{1}\right) & =\inf \left\{\int_{T}\|q(t)-r(t)\| d t: r(\cdot) \in S_{R_{n}\left(\cdot, y_{m}\right)}^{1}\right\} \\
& =\int_{T} \inf \left[\|q(t)-u\|: u \in R_{n}\left(t, y_{m}\right)\right] d t=\int_{T} d\left(q(t), R_{n}\left(t, y_{m}\right)\right) d t \\
& \Rightarrow \limsup _{m \rightarrow \infty} \int_{T} d\left(q(t), R_{n}\left(t, y_{m}\right)\right) d t \leqq \int_{T} \limsup _{m \rightarrow \infty} d\left(q(t), R_{n}\left(t, y_{m}\right)\right) d t .
\end{aligned}
$$

From Theorem 2.2. (i) of Tsukada [18] (Tsukada states his results for 
convex sets. However a look at his proof can convince the reader that it is also true for nonconvex ones), we have

$$
\limsup _{m \rightarrow \infty} d\left(q(t), R_{n}\left(t, y_{m}\right)\right) \leqq d\left(q(t), \text { s-liminf } R_{m \rightarrow \infty}\left(t, y_{m}\right)\right)
$$

But we have already seen that $R_{n}(t, \cdot)$ is lower semicontinuous. So we have:

$$
\begin{gathered}
R_{n}(t, y) \leqq \mathrm{s}-\liminf _{m \rightarrow \infty} R_{n}\left(t, y_{m}\right) \\
\Rightarrow d\left(q(t), \operatorname{s}_{m \rightarrow \infty}-\liminf _{n}\left(t, y_{m}\right)\right) \leqq d\left(q(t), R_{n}(t, y)\right) \\
\Rightarrow \limsup _{m \rightarrow \infty} d\left(q, S_{R_{n}\left(\cdot, y_{m}\right)}^{1}\right) \leqq \int_{T} d\left(q(t), R_{n}(t, y)\right) d t=d\left(q, S_{R_{n}(\cdot, y)}^{1}\right) .
\end{gathered}
$$

Since $\quad$ s-liminf ${ }_{m \rightarrow \infty} S_{R_{n}\left(\cdot, y_{m}\right)}^{1}=\left\{p \in L^{1}(T, X): \lim _{m \rightarrow \infty} d\left(p, S_{R_{n}\left(\cdot, y_{m}\right)}^{1}\right)=0\right\} \quad$ (see Kuratowski $[9$, p. 333]), from the above inequality we deduce that:

$$
\begin{gathered}
\quad S_{R_{n}(\cdot, y)}^{1} \cong \mathrm{s}-\liminf _{m \rightarrow \infty} S_{R_{n}\left(\cdot, y_{m}\right)}^{1} \\
\Rightarrow H_{n}(y) \cong \mathrm{s}-\liminf _{m \rightarrow \infty} H_{n}\left(y_{m}\right) \\
\Rightarrow H_{n}(\cdot) \text { is lower semicontinuous for all } n \geqq 1 .
\end{gathered}
$$

Step 6. We can find $x_{n}(\cdot) \in P$ such that $\left\|\dot{v}_{n}(t)-\dot{x}_{n}(t)\right\| \leqq d\left(\dot{v}_{n}(t)\right.$, $F\left(t, x_{n}(t)\right)+1 / n$ a.e.

We saw in Step 2 that $W$ is compact in $C(T, X)$. Also in Step 5 we proved that $H_{n}(\cdot)$ is lower semicontinuous on $W$. So we can apply the selection theorem of Fryszkowski [6] and get continuous functions $h_{n}: W \rightarrow$ $L^{1}(T, X)$ such that $h_{n}(y) \in H_{n}(y)$ for all $y(\cdot) \in W \Rightarrow h_{n}(y)(t) \in R_{n}(t, y)$ a.e. $\Rightarrow$ for all $n \geqq 1 \quad h_{n}(y)(t) \in F(t, y(t))$ a.e. and $\left\|\dot{v}_{n}(t)-h_{n}(y)(t)\right\| \leqq d\left(\dot{v}_{n}(t)\right.$, $F(t, y(t)))+1 / n$ a.e. Consider the integral operator $\Phi_{n}: W \rightarrow W$ defined by

$$
\Phi_{n}(y)(t)=x_{0}+\int_{0}^{t} h_{n}(y)(s) d s, \quad t \in T .
$$

Clearly $\Phi_{n}(\cdot)$ is continuous. Using the Schauder fixed point theorem we can find $\left\{x_{n}(\cdot)\right\}_{n \geqq 1} \subseteq W$ such that $\Phi\left(x_{n}\right)=x_{n}$. Hence we have

$$
x_{n}(\cdot) \in P \text { and }\left\|\dot{v}_{n}(t)-\dot{x}_{n}(t)\right\| \leqq d\left(\dot{v}_{n}(t), F(t, x(t))\right)+\frac{1}{n} \quad \text { a.e. }
$$

Step 7. We will show that $x_{n}(\cdot) \stackrel{C_{X}(T)}{\longrightarrow} x(\cdot)$ as $n \rightarrow \infty$.

Since $\left\{x_{n}(\cdot)\right\}_{n \geq 1} \subseteq W$ and $W$ is compact in $C_{X}(T)$, by passing to a subsequence if necessary, we may assume that $x_{n}(\cdot) \stackrel{C_{X}(T)}{\longrightarrow} y(\cdot) \in W$. Let

$$
k_{n}(t)=\left\|v_{n}(t)-x_{n}(t)\right\| \text {. }
$$

Then $k_{n}(t) \rightarrow k(t)=\|x(t)-y(t)\|$ for all $t \in T$. Also using the hy- 
pothesis 2 we have:

$\dot{k}_{n}(t) \leqq\left\|\dot{v}_{n}(t)-\dot{x}_{n}(t)\right\| \leqq d\left(\dot{v}_{n}(t), F\left(t, x_{n}(t)\right)\right)+\frac{1}{n} \leqq w\left(t,\left\|x_{n}(t)-x(t)\right\|\right)+\frac{1}{n} \quad$ a.e.

Take $B>0$ stch that $\left\|x_{n}(t)-x(t)\right\|<B$ for all $n \geqq 1$ and all $t \in T$. From the Scorza-Dragoni theorem we know that we can find a measurable set $T_{m}$ with $\lambda\left(T \backslash T_{m}\right)<1 / m$ such that $\left.w\right|_{T_{m} \times[0, B]}$ is uniformly continuous. So given $\delta>0$ we can find $n_{m}(\delta)$ such that for $n \geqq n_{m}$ and for all $t \in T_{m}$ we have:

$$
\begin{gathered}
w\left(t,\left\|x_{n}(t)-x(t)\right\|\right) \leqq w(t,\|y(t)-x(t)\|)+\delta \\
\Rightarrow \dot{k}_{n}(t) \leqq w(t, k(t))+\frac{1}{n}+\delta \quad \text { a.e. on } T_{m}, \quad m \geqq 1 .
\end{gathered}
$$

Also from Theorem 4.1 of Davy [4] we have that:

$$
\dot{k}(t) \in \bigcap_{i=1}^{\infty} \text { clconv } \bigcup_{n=i}^{\infty} \dot{k}_{n}(t) \text { a.e. }
$$

So at the limit as $n \rightarrow \infty$ we get that:

$$
\dot{k}(t) \leqq w(t, k(t))+\delta \quad \text { a.e. on } T_{m}, \quad m \geqq 1 .
$$

Recall that $\delta>0$ was arbitrary. Hence finally we can write that

$$
\dot{k}(t) \leqq w(t, k(t)) \text { a.e. on } T_{m}, \quad m \geqq 1 \text {. }
$$

But $\lambda\left(T \backslash \cup_{m=1}^{\infty} T_{m}\right)=0$. Therefore $\dot{k}(t) \leqq w(t, k(t))$ a.e. Since $k(0)=\| y(0)-$ $x(0)\|=\| x_{0}-x_{0} \|=0$ and $w(\cdot, \cdot)$ is a Kamke function, we have that $k(\cdot) \equiv$ $0 \Longrightarrow y(t)=x(t)$ for all $t \in T \Longrightarrow x_{n}(\cdot) \stackrel{C_{X}(T)}{\longrightarrow} x(\cdot)$. Because $x_{n}(\cdot) \in P$, the proof of the theorem is completed. $\quad$ q.e.d.

REMARK. When $X=\boldsymbol{R}^{n}$ we get an improved version of Theorem 1 of Pianigiani [11], since we do not require the orientor field to be continuous in the time variable $t$. Pianigiani assumed that $F(\cdot, \cdot)$ was jointly continuous.

\section{REFERENCES}

[1] N. Ahmed ANd K. Teo, Optimal Control of Distributed Parameter Systems, North-Holland New York, Oxford, 1981

[2] C. Castaing and M. Valadier, Convex Analysis and Measurable Multifunctions, Lecture Notes in Math. 580, Springer-Verlag, Berlin, Heidelberg, New York, 1977.

[ 3 ] F. ClaRke, Optimization and Nonsmooth Analysis, Wiley, New York, 1983.

[4] J. Davy, Properties of the solution set of a generalized differential equation, Bull. Austral. Math. Soc., 6 (1972), 379-398.

[5] A.F. Filippov, Classical solutions of differential equations with multivalued righthand side, SIAM J. Control, 5 (1967), 609-621.

[6] A. Fryszkowski, Continuous selections for a class of nonconvex multivalued maps, Studia 
Math. 76 (1983), 163-174.

[7] E. Klein and A. Thompson, Theory of Correspondences, Wiley, New York, 1984.

[8] I. Kluvanek and G. KNowles, Vector Measures and Control Systems, North Holland, Amsterdam, Oxford, 1976.

[9] K. Kuratowski, Topology I, Academic Press, New York, 1966.

[10] U. Mosco, Convergence of convex sets and of solutions of variational inequalities Advances in Math. 3 (1969), 510-585.

[11] G. Pianigiani, On the fundamental theory of multivalued differential equations, J. Differential Equations, 25 (1977), 30-38.

[12] A. PLISS, Trajectories and quasitrajectories of an orientor field, Bull. Polish Academy of Sciences, 10 (1962), 529-531.

[13] N.S. Papageorgiou, On the theory of Banach space valued multifunctions. Part I: Integration and conditional expectation, J. Multivariate Anal, 17 (1985), 185-206.

[14] N. S. Papageorgiou, Random differential inclusions in Banach spaces, J. Differential Equations, 65 (1986), 287-303.

[15] R. T. RockAfellaR, Integral functionals, normal integrands and measurable selections, in Nonlinear Operators and the Calculus of Variations, L. Waelbroeck, Ed., Lecture Notes in Math. 543, Springer-Verlag, Berlin, Heidelberg, New York, 1976, 157-207.

[16] G. SALINETTI AND R. WETS, On the relation between two types of convergence for convex functions, J. Math. Anal. Appl. 60 (1977), 211-226.

[17] A. Tolstonogov, On differential inclusions in Banach spaces and continuous selections, Soviet Math. Doklady, 20 (1979), 186-190.

[18] M. TsukadA, Convergence of best approximations in a smooth Banach space, J. Approx. Theory, 40 (1984), 301-309.

[19] D. WAGNER, Survey of measurable selection theorems, SIAM Jour. Control Optim, 15 (1977), 859-903.

[20] T. WAŻEWSKI, Sur une generalisation de la notion des solutions d'une equation au contingent, Bull. Polish Academy of Sciences, 10 (1962), 11-15.

[21] R. WETs, A formula for the level sets of epi-limits and some applications, in Mathematical Theories of Optimization, J. Cecconi and T. Zolezzi, Eds., Lecture Notes in Math. 976, Berlin, Heidelberg, New York, 1983, 256-268.

University of Thessaloniki and Department of Mathematics

School of TeChNOLOGY

University of CALIFORNia

Mathematics Division

DAvis, California 95616

THESSALONIKI 54006

U.S.A.

Greece 
